\title{
Meta
}

Journal des traducteurs

Translators' Journal

\section{Les terminologies spécialisées : approche quantitative et logico-sémantique (Deuxièmes entretiens du Centre Jacques-Cartier, Montréal, 12-14 octobre 1988)}

\section{Henri Béjoint}

Volume 34, numéro 2, juin 1989

URI : https://id.erudit.org/iderudit/002023ar

DOI : https://doi.org/10.7202/002023ar

Aller au sommaire du numéro

Éditeur(s)

Les Presses de l'Université de Montréal

ISSN

0026-0452 (imprimé)

1492-1421 (numérique)

Découvrir la revue

Citer cette note

Béjoint, H. (1989). Les terminologies spécialisées : approche quantitative et logico-sémantique (Deuxièmes entretiens du Centre Jacques-Cartier, Montréal, 12-14 octobre 1988). Meta, 34(2), 316-317. https://doi.org/10.7202/002023ar d'utilisation que vous pouvez consulter en ligne. 
BLOC-NOTES

\section{LES TERMINOLOGIES SPÉCIALISÉES : APPROCHE QUANTITATIVE ET LOGICO-SÉMANTIQUE \\ (Deuxièmes entretiens du Centre Jacques-Cartier, Montréal, 12-14 octobre 1988)}

Les Deuxièmes entretiens du Centre JacquesCartier, organisés conjointement par le Centre Jacques-Cartier, basé à Lyon, et l'université de Montréal, se sont déroulés à Montréal du 12 au 24 octobre 1988 (les Premiers entretiens avaient eu lieu à Lyon en juin 1987). Ces Deuxièmes entretiens comprenaient 14 colloques sur des sujets très divers : «Le médicament vétérinaire», «Cardiologie», «Mathématiques discrètes», «Démographie intergénérationnelle», «Urbanisme et patrimoine», «Structure élémentaire de la matière hadronique», etc.

Le $8^{\mathfrak{e}}$ de ces colloques, placé sous la responsabilité scientifique d'André Clas et Jean Baudot pour l'Université de Montréal et de Philippe Thoiron et Henri Béjoint pour l'Université Lumière Lyon 2, portait le titre «Les terminologies spécialisées :approches quantitative et logico-sémantique». Pendant les deux journées de travail, 17 orateurs se sont exprimés. Il ne peut pas être question de résumer de façon satisfaisante en quelques lignes des exposés qui pour la plupart correspondent à un travail de plusieurs années. Pardon, donc, à leurs auteurs, qui me liront ici et qui sans doute me trouveront bien injuste. Qu'ils se consolent en pensant que leur travail sera bientôt intégralement publié dans $M E T A$ !

L'exposé de Michel Le Guern («Sur les relations entre terminologie et lexique») était une tentative originale de différencier les deux domaines du terme et du mot, l'un appartenant à une logique extensionnelle (le terme), et l'autre à une logique intensionnelle (le mot), l'un étant en rapport direct avec la référence, par l'intermédiaire de la syntaxe (le terme), l'autre ne l'étant pas (le mot), l'un ayant un concept (le terme), l'autre ayant un signifié (le mot). L'intervention de Jacques Lethuillier («Réflexions sur la synonymie en langues de spécialité») rappelait les grands principes de la terminologie, et replaçait, à l'aide de nombreux exemples concrets, la notion de synonymie dans le contexte général de l'efficacité de la communication. Henri Béjoint ( $\ll$ À propos de la monosémie en terminologie») montrait à quel point le concept de monosémie est discutable dans une terminologie qui se veut onomasiologique, et combien ce concept est difficile à évaluer précisément si l'on accepte l'idée d'une terminologie sémasiologique.

Geneviève Mareschal («Repérage d'unités terminologiques dans le contexte de l'enseignement de la traduction spécialisée») reprenait l'ensemble des problèmes posés par l'aspect terminologique de l'enseignement de la traduction spécialisée. L'exposé de Jean-Claude Boulanger ( $\ll$ Le statut du syntagme dans les dictionnaires de langue monolingues») montrait que le traitement des syntagmes dans les dictionnaires généraux est différent selon les dictionnaires. Dorothy Nakos («Étude comparée des modes de formation des lexies complexes dans deux domaines différents») montrait à l'aide de nombreux exemples comment deux disciplines différentes peuvent avoir recours à des procédés différents (au moins statistiquement) pour la création de nouveaux termes.

L'exposé de Richard Bouché («Le statut du mot clé. Une nouvelle approche des bases de données textuelles») montrait comment on pouvait expliquer les idées de Michel Le Guern à un travail pratique sur l'indexation automatique dans une base de données textuelles. Il s'agit d'utiliser un analyseur syntaxique pour isoler les syntagmes nominaux qui seront retenus comme descripteurs. Richard Kittredge («Analyse sémantique et transfert lexical pour la traduction automatique»), spécialiste de traduction automatique, doit faire face à certains des problèmes rencontrés par les gestionnaires de bases de données. Il montrait, avec des exemples très concrets, comment une analyse purement formelle des discours est incapable de rendre compte de certains faits : l'exemple de EN replace oil filter, équivalent soit de FR replacer le filtre à huile, soit de FR remplacer le filtre à huile, était assez éloquent. On peut rapprocher ses conclusions de celles de Christine Portelance («Terminologies: syntagmes et paradigmes»), qui montrait qu'une analyse purement syntagmatique ne peut pas rendre compte de tout, et qu'il faut parfois avoir recours à des critères sémantiques (en l'occurrence «paradigmatiques») pour désambiguiser un syntagme : par exemple (courant présumé) établi ou courant (présumé établi). 
Louise Larivière («Vers un produit unifié en terminologie et en documentation: le thésaurus terminologique»), comme son titre l'indique clairement, plaidait pour la création de thésaurus terminologiques et en montrait l'utilité pour la science documentaire. L'exposé d'Agnès Kukulska-Hulme ( «L'organisation conceptuelle des dictionnaires automatiques pour textes techniques») examinait comment une base de données terminologiques peut aider à la traduction spécialisée. Parmi les différentes organisations possibles d'une telle base de données, il semble que ce soit celle qui se fonde sur les collocations privilégiées de chaque terme qui soit la plus utile.

Jacques Jansen («Apport contrastif des dictionnaires généraux de la langue au problème de l'indexation automatique dans le discours technicoscientifique») rendait compte des travaux réalisés depuis plusieurs années par l'équipe de Liège à partir notamment des bandes magnétiques qui contiennent la version pré-publication du dictionnaire monolingue Longman (LDOCE). L'exposé portait sur une exploitation quantitative des marques d'usage dans les dictionnaires (LDOCE et le Robert/Collins) et dans un corpus de référence, l'objectif étant de formaliser la reconnaissance par l'homme du degré de spécialisation d'un texte donné.

Les quatre exposés suivants, bien que très différents, abordent fondamentalement ce même problème des degrés de spécialisation d'un texte scientifique. Malcolm Clay («Approche quantitative de l'étude des marqueurs du niveau de spécialisation dans des textes scientifiques anglais») fait intervenir la notion de choix (lexicaux et syntaxiques) de l'énonciateur. Cette approche «relative» permet des conclusions plus intéressantes qu'une approche «absolue», simple comptage de formes. Philippe Thoiron ( $\ll \mathrm{La}$ topographie des termes»), par une approche statistique, montrait que, davantage que sa simple fréquence dans un texte, c'est la répartition d'un terme $X$ qui permet de caractériser le texte et de préciser les rapports entre $X$ et ce texte : les termes «génériques» sont également répartis, alors que les termes «spécifiques» sont concentrés dans certaines parties du texte, qui peuvent dès lors être extraites automatiquement. Nathan Ménard («Mesure des relations lexico-sémantiques dans les textes scientifiques : problèmes méthodologiques») travaille sur la notion de cohésion à l'intérieur d'un texte. À l'aide de trois textes témoins (Einstein, Reeves et Albert Camus), il montre que les différents types de cohésion qu'il a préalablement définis sont représentés différemment selon la nature du texte. D'une façon générale, la cohésion est plus forte dans les textes scientifiques que dans la prose d'un romancier par exemple. Enfin, l'exposé de Sylvia Pavel («Mise en évidence des niveaux linguistiques dans la terminologie de l'intelligence artificielle») développait l'idée que la prise en compte des niveaux de langue en terminologie est non seulement intéressante, mais indispensable, surtout dans une discipline en devenir comme l'IA où le terminologue ne peut se contenter d'explorer la langue standard.

Pour conclure, Pierre Auger ( $«$ Terminologies et industries de la langue») faisait le bilan de ce qui se fait actuellement en terminologie, au Canada bien sûr mais aussi ailleurs dans le monde : ce qui est d'ores et déjà acquis, ce qu'on peut entrevoir dans un avenir proche, et les projets à plus long terme.

En deux jours, les questions fondamentales de la terminologie ont été soulevées et leurs différentes solutions ont été réévaluées, de façon souvent contradictoire et donc riche d'enseignements, en fonction de leur efficacité pratique dans les problèmes de la construction de thésaurus, de l'indexation automatique et du repérage des niveaux de spécialisation des discours techno-scientifiques. Les Actes seront publiés très prochainement dans META. Abonnezvous! Abonnez-vous! Ou réabonnez-vous!

HENRI BÉJOINT

Université Lumière Lyon 2, Lyon, France 